Kastamonu Civarındaki Evcil Sığırların (Bos taurus taurus) İşkembesinde Tespit Edilen İki Siliyat Türü Entodinium palmare ve E. okoppensis (Protista: Ciliophora: Ophryoscolecidae) Hakkında

\title{
About Ciliates Entodinium palmare and E. okoppensis (Ciliophora: Ophryoscolecidae) in the Rumen of Domestic Cattles (Bos taurus taurus) in the vicinity of Kastamonu
}

\section{Gözde Gürelli}

Kastamonu Üniversitesi Fen-Edebiyat Fakültesi, Biyoloji Anabilim Dalı, Kastamonu, Türkiye

\begin{abstract}
ÖZET
Amaç: Bu çalışmanın amacı Kastamonu civarında yaşayan evcil sığıların işkembesinde bulunan siliyat türleri Entodinium palmare ve E. okoppensis'in sitolojik özelliklerini tespit etmek, türlerin morfolojik karakterlerini orijinal tanımlarıyla karşılaştırarak benzerlik ve farklılıkları tartışmaktır.

Yöntemler: Yirmi dört sığırdan elde edilen örnekler \%10'luk formalinle tespit edildikten sonra metil formalin salin (MFS) ile boyanmıştır.

Bulgular: Ülkemizdeki sığılardan elde edilen bireylere ait ölçümler, morfolojik karakterler ve biyometrik veriler orijinal tanımlarlarla benzer bulunmuştur Sonuç: E. palmare ve E. okoppensis ülkemizdeki sığırlardan ilk kez dünyadaki sığırlardan ise ikinci kez bu çalışmayla rapor edilmiştir. E. palmare ve E. okoppensis ülkemiz sığırlarında oldukça düşük bir görülme sıklığı ve bulunma oranına sahiptirler. Bu durumun nedenleri olarak sığırların beslenme alışkanlıkları, beslenme sıklıkları, işkembenin fizyolojik durumu veya türler arasında görülen rekabetin önemli olabileceği sonucuna varılmıştır. (Turkiye Parazitol Derg 2012; 36: 228-31)
\end{abstract}

Anahtar Sözcükler: Entodinium palmare, E. okoppensis, işkembe, siliyat, sığır

Geliş Tarihi: 03.09.2012

Kabul Tarihi: 10.10 .2012

\section{ABSTRACT}

Objective: The aim of this study was to determine the cytological features of the rumen ciliates Entodinium palmare and E. okoppensis living in the rumen of domestic cattle in the vicinity of Kastamonu, compare the morphological characters of species with their original descriptions and discuss the similarities and differences.

Methods: The specimens were obtained from 24 cattle, fixed with 10\% formalin and stained with methylgreen formalin saline (MFS) solution Results: Specimens were measured from cattle in our country, and were found to be similar to the original description on the basis of morphological characteristics and biometric data.

Conclusion: With this investigation, E. palmare and E. okoppensis were firstly detected from our cattle and were secondly detected from cattle throughout the world. E. palmare and E. okoppensis have low frequency appearance and percentage composition in our cattle. This study concluded that the feeding habits and feeding frequencies of cattle, the physiological conditions of rumen or competition between species can be important reasons for this situation. (Turkiye Parazitol Derg 2012; 36: 228-31)

Key Words: Entodinium palmare, E. okoppensis, rumen, ciliate, cattle

\section{GiRiş}

Dünyanın farklı bölgelerinde yaşayan herbivor konakların işkembesindeki siliyat türlerinin belirlenmesi siliyatların coğrafi varyasyonları, konakların beslenme alışkanlıkları ve siliyatların filogenileri hakkında bilgi sağlamaktadır (1-5).
Işsembe siliyat tür kompozisyonu farklı konaklar arasında, hatta aynı konak türün farklı coğrafik alanlarında yaşayan bireylerinde farklı olabilir (6-8).

Entodinium palmare ve E. okoppensis Ophryoscolecidae familyasına ve Entodiniomorphida ordosuna dahil endo-

Yazışma Adresi / Address for Correspondence: Dr. Gözde Gürelli, Kastamonu Üniversitesi Fen-Edebiyat Fakültesi, Biyoloji Anabilim Dalı, Kastamonu, Türkiye Tel: +90 3662801906 E-posta: ggurelli@yahoo.com doi:10.5152/tpd.2012.55 
kommensal siliyatlardır (9). E. palmare ilk kez 2009 yılında Tanzanya'daki kısa boynuzlu Zebu sığırlarından, E. okoppensis ise ilk kez 1990 yılında Japonya'daki Holstein sığırlardan tanımlanmıştır $(10,11)$.

Bu çalışmanın amacı Kastamonu civarında yaşayan evcil sığırların (Bos taurus taurus L.) işkembesinde bulunan siliyat türleri E. palmare ve E. okoppensis'in sitolojik özelliklerini tespit etmek, türlerin morfolojik karakterlerini orijinal tanımlarıyla karşılaştırarak benzerlik ve farklılıkları tartışmaktır.

\section{YÖNTEMLER}

Işsembe örnekleri Kastamonu ve civarındaki çeşitli mezbahalardaki 24 sığırdan Mart 2012 ve Haziran 2012 tarihleri arasında alınmıştır. Örnekler siliyatların bozulmasını engellemek için hemen \%10'luk formalinle tespit edilmiştir. Laboratuara getirildikten sonra ağ gözü sayısı 50 ve açıklığı 582.5 m olan hücre ayrıştırma eleğinden (Sigma) geçirilerek süzülmüştür. Daha sonra elde edilen depo örneklerden küçük cam tüplere ölçekli pipet yardımıyla bir miktar alınarak, MFS ile geçici preparatlar hazırlanmişıtr (12-14).

Siliyatların orientasyonu için Dogiel'den yararlanılmıştır (15). Bu orientasyon sisteminde, öncelikle hücrenin anteriyör-posteriyör yönelimi saptanır. Sitoproktun bulunduğu taraf daima posteriyör, karşı taraf ise anteriyördür. Nukleus apareyine en yakın vücut kısmı dorsal karşı tarafı hücrenin ventralidir. Sağ ve sol taraflar ise organizmanın dorsal tarafının gözlemcinin sırt tarafıyla aynı doğrultuda olduğu düşünülerek saptanır.

Işık mikroskobunda inceleme, fotoğraf çekimi ve örneklere ait ölçümler Leica DM 3000 görüntüleme sistemiyle gerçekleştirilmiştir. Sınıflandırma ve tür tayini Lynn (9), Mishima ve ark. (10) ve Ito ve Imai (11) dayandırılarak verilmiştir. Morfolojik karakterlerle ilgili istatistiksel verilerin elde edilmesinde SPSS (Vers. 10.0) istatistik programı kullanılmıştır.

\section{BULGULAR}

\section{E. palmare Mishima et al. (10)}

Vücut ovoittir, yanlardan basıktır, ön uca doğru hafifçe daralır. Vücudun ön ucunda ileri-geri çekilebilen (retraktil) oral sil zonunun belirgin bir dudağı bulunur. Vücudun posteriyör dorsal parçası sağ tarafta konkavdır ve bu parça dorsal taraftan bakıldığında sağ uca doğru eğimlenir. Yüzeysel ve geniş oluk vücudun sol yüzeyinde boyuna uzanır (sol tarafta hücrenin uzun eksenine paralel uzanır). Vestibulum huni şekillidir ve dorsale yönelir. Sitoprokt konkavlığın ventral kenarında ve arka uçtadır. Makronukleus çubuk şekillidir ve vücudun dorsal tarafındadır. Küçük ovoid mikronukleus makronukleusun ventral sol tarafına yerleşmiştir. Tek kontraktil vakuol mikronukleusun ön tarafında, makronukleusun ön ucunun sol ventral tarafinda bulunur (Şekil 1).

E. palmare incelenmiş olan 24 sığırın 1'inde gözlemlenmiştir. Görülme sıklığı \%4.2'dir. Sığır 10'da bulunma oranı \%2.4'tür.

E. palmare'ye ait sığırlarımızdan saptanan morfometrik değerler Tablo 1'de verilmiştir. Tablo 2'de ise E. palmare'nin vücut ölçümleri orijinal tanımlamadaki örnekle karşılaştırılmıştır.

\section{Entodinium okoppensis Ito ve Imai (11)}

Vücut dikdörtgenle kare arası değişir. Vücudun arka ucunda çeşitli büyüklükte 1-3 kaudal ışın veya lob bulunur. Vücudun ön ucu düz veya konkavdır. Ön dudak, oral siller geri çekildiğinde hemen hemen hiç görülmez. Vestibulum geniş ve huni şekillidir. Dikey olarak uzanır fakat hafifçe sola yönelir. Sitoprokt vücudun posteriyör ucunda orta sol taraftadır. Makronukleus düz ve ince çubuk şekillidir, vücut uzunluğunun 4/5'i kadar uzunluktadır. Vücudun sağ-dorsal tarafına yerleşmiştir. Makronukleusun ön ucu düz, arka ucu ise yuvarlaktır. Oval mikronukleus makronukleusun orta sol kenarında bulunur. Kontraktil vakuol makronukleusun tam önünde ve biraz üzerindedir.

Rumen sıvısı örneklerinde bu türe ait 2 morfotipi tespit edilmiştir.

\section{E. okoppensis m. okoppensis (Şekil 2a)}

Üç kaudal ışın bulunur. Sağ olan sivri veya küt ışın şeklindedir, bazen dış tarafa doğru bükülür, sol alt ve sol üstteki kaudal çıkıntılar sivri ışınlar şeklindedir.

\section{E. okoppensis m. bifidum (Şekil 2b)}

Sadece sol tarafta aynı uzunlukta 2 kaudal ışın mevcuttur.

E. okoppensis incelenmiş olan 24 sığıın $1^{\prime}$ inde gözlemlenmiştir. Görülme sıklığı \%4.2'dir. Sığır 15'de bulunma oranı \%6.3'tür.

E. okoppensis'e ait sığırlarımızdan saptanan morfometrik değerler Tablo 3'te verilmiştir. Tablo 4'te ise E. okoppensis'in vücut ölçümleri orijinal tanımlamadaki örnekle karşılaştırılmıştır.
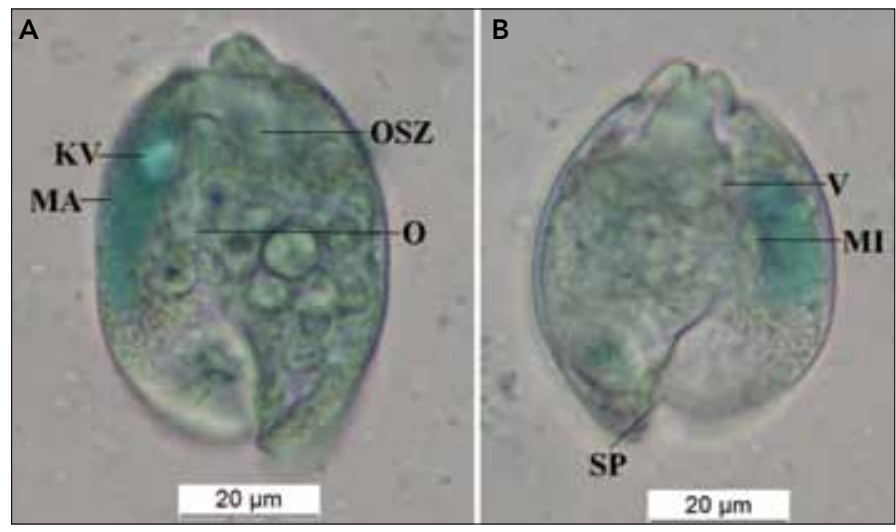

Şekil 1. E. palmare (A) sağdan, (B) soldan

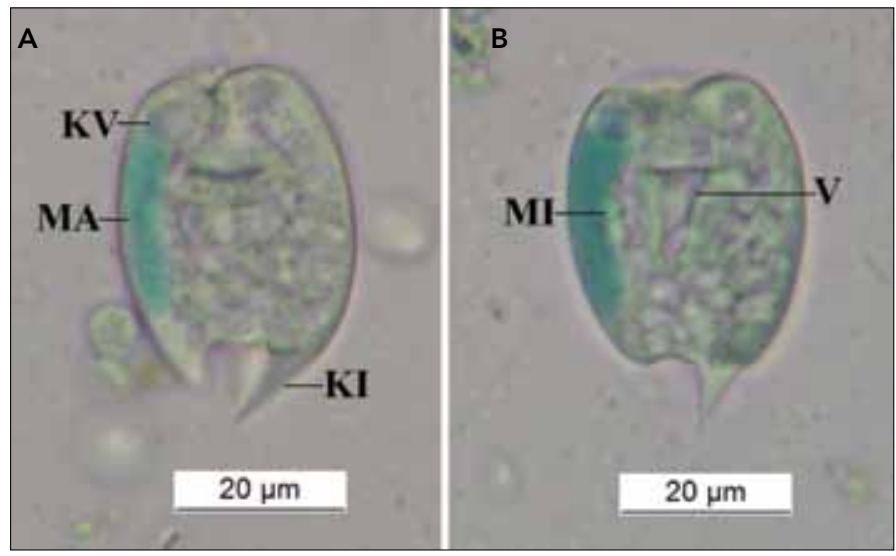

Şekil 2. E. okoppensis m. okoppensis (a) sağdan, E. okoppensis m. bifidum (b) sağdan 
Tablo 1. E. palmare'ye ait ölçüm değerleri ve bu karakterlere ilişkin biyometrik veriler

\begin{tabular}{|l|c|c|c|c|}
\hline & \multicolumn{4}{|c|}{ Entodinium palmare (n=25) } \\
\hline Karakterler & Ekstr. & Ort. & SD & SE \\
\hline$[\mathrm{U}]$ & $42.2-68.3$ & 60.0 & 7.2 & 1.4 \\
\hline$[\mathrm{G}]$ & $37.1-52.4$ & 42.9 & 4.4 & 0.9 \\
\hline$[\mathrm{MaU}]$ & $17.5-35.5$ & 27.6 & 5.7 & 1.1 \\
\hline$[\mathrm{MaG}]$ & $6.6-10.4$ & 7.9 & 1.0 & 0.2 \\
\hline$[\mathrm{U} / \mathrm{G}]$ & $1.1-1.6$ & 1.4 & 0.1 & $<0.1$ \\
\hline$[\mathrm{MaU} / \mathrm{MaG}]$ & $2.0-5.1$ & 3.6 & 0.9 & 0.2 \\
\hline$[\mathrm{U} / \mathrm{MaU}]$ & $1.7-3.0$ & 2.2 & 0.3 & 0.1 \\
\hline
\end{tabular}

n: Örnek sayısı, U: Hücre uzunluğu, G: Hücre genişliği, MaU: Makronukleus uzunluğu, MaG: Makronukleus genişliği, Ekstr.: Ekstrem değerler, Ort.: Aritmetik ortalama, SD: Standart sapma, SE: Standart hata

Tablo 2. E. palmare'nin vücut ölçümleri ile ilgili bulguların orijinal tanımlamadaki örnekle karşılaştırılması

\begin{tabular}{|l|c|c|c|c|c|}
\hline Kaynak & Ülke & Konak & [U] & [G] & [U/G] \\
\hline 10 & Tanzanya & Sığır & $44.4 \pm 4.7$ & $31.9 \pm 4.9$ & $1.4 \pm 0.3$ \\
\hline Bu çalışma & Türkiye & Sığır & $60.0 \pm 7.2$ & $42.9 \pm 4.4$ & $1.4 \pm 0.1$ \\
\hline 10: Mishima ve ark.
\end{tabular}

Tablo 3. E. palmare'e ait ölçüm değerleri ve bu karakterlere ilişkin biyometrik veriler

\begin{tabular}{|l|c|c|c|c|}
\hline & \multicolumn{5}{|c|}{ Entodinium palmare (n=25) } \\
\hline Karakterler & Ekstr. & Ort. & SD & SE \\
\hline$[\mathrm{U}]$ & $22.5-39.6$ & 33.2 & 4.7 & 0.9 \\
\hline$[\mathrm{G}]$ & $21.4-32.2$ & 27.5 & 3.0 & 0.6 \\
\hline$[\mathrm{KIU}]$ & $3.2-10.9$ & 5.5 & 1.5 & 0.3 \\
\hline$[\mathrm{MaU}]$ & $17.6-34.7$ & 23.9 & 4.1 & 0.8 \\
\hline$[\mathrm{MaG}]$ & $3.9-7.1$ & 5.1 & 0.9 & 0.2 \\
\hline$[\mathrm{U} / \mathrm{G}]$ & $0.9-1.4$ & 1.2 & 0.1 & $<0.1$ \\
\hline$[\mathrm{MaU} / \mathrm{MaG}]$ & $3.3-6.3$ & 4.8 & 0.8 & 0.2 \\
\hline$[\mathrm{U} / \mathrm{MaU}]$ & $1.1-1.7$ & 1.4 & 0.2 & $<0.1$ \\
\hline $\begin{array}{l}\text { n: Örnek sayısı, U: Hücre uzunluğu, G: Hücre genişliği, KIU: Kaudal ışın } \\
\text { uzunluğu, MaU: Makronukleus uzunluğu, MaG: Makronukleus genişliği, } \\
\text { Ekstr:: Ekstrem değerler, Ort.: Aritmetik ortalama, SD: Standart sapma, } \\
\text { SE: Standart hata }\end{array}$ \\
\hline
\end{tabular}

Tablo 4. E. okoppensis'in vücut ölçümleri ile ilgili bulguların orijinal tanımlamadaki örnekle karşılaştırılması

\begin{tabular}{|l|c|c|c|c|c|}
\hline Kaynak & Ülke & Konak & {$[\mathbf{U}]$} & {$[\mathbf{G}]$} & {$[\mathbf{U} / \mathbf{G}]$} \\
\hline 11 & Japonya & Sığır & $35.7 \pm 6.3$ & $26.2 \pm 3.0$ & $1.4 \pm 0.2$ \\
\hline Bu çalışma & Türkiye & Sığır & $33.2 \pm 4.7$ & $27.5 \pm 3.0$ & $1.2 \pm \_0.1$ \\
\hline 11: Ito ve ark.
\end{tabular}

\section{TARTISSMA}

E. palmare ve E. okoppensis bu çalışmayla ülkemiz sığırlarından ilk kez, dünyadan ise ikinci kez rapor edilmiştir. Araştırmada sığırlarımızdan ölçülen örnekler, morfolojik karakterler ve biyometrik veriler bakımından orijinal tanımlarıyla benzer bulunmuştur.
Ülkemiz sığırlarında belirlenen E. palmare'nin vücut uzunluğu ve genişliği Tanzanya'daki Zebu sığırlarından belirlenen değerlere göre büyük olmasına rağmen, uzunluğun genişliğe oranı aynıdır (Tablo 2). Ülkemiz sığırlarından belirlenen E. okoppensis'in vücut uzunluğu ve genişliği Japonya'daki örneklerle hemen hemen aynıdır (Tablo 4).

Sığırlar üzerinde daha önce ülkemiz ve dünyada yapılan çeşitli çalışmalarda bu türlere rastlanmaması ve sadece E. palmare'nin Tanzanya'daki ve E. okoppensis'in Japonya'daki sığırlardan tespit edilmesi ve ülkemizde Kastamonu civarındaki sığırlardan rastlanması oldukça şaşırtıcıdır (2, 4-6, 16-28). Japonya'daki sığırlardan E. okoppensis'in 4 morfotipi rapor edilmesine rağmen, ülkemiz sığırlarında sadece 2 morfotipi tespit edilmiştir. Ayrıca E. okoppensis'in bir morfitipi E. okoppensis m. cameli Çin'deki develerden kaydedilmiştir (11). Bununla birlikte, sığırlardan tespit edilen 4 morfotip develerde gözlenmemiştir (29).

\section{SONUÇ}

E. palmare ve E. okoppensis oldukça düşük bir görülme sıklığı ve bulunma oranına sahiptir. Bu durumun nedenleri olarak sığırların beslenme alışkanlıkları, beslenme sıklıkları, işkembenin fizyolojik durumu veya türler arasında görülen rekabetin önemli olabileceği sonucuna varılmıştır.

E. palmare'nin Tanzanya ve Türkiye gibi farklı kıtalarda bulunan ülkelerin işkembe siliyat faunasında yer alması, E. okoppensis'in ise aynı kıtada fakat birbirine oldukça uzak coğrafi bölgelerden kaydedilmesi herbivorların işkembe siliyat faunası üzerine daha fazla çalışmanın yapılması gerektiğinin göstergesidir. Böylece farklı bölgelerde yaşayan farklı konakların incelenmesiyle bu türün kıtalararası nasıl geçiş gösterdiğinin anlaşılması açısından önemlidir.

\section{Teşekkür}

Örneklerin temininde ve taşınmasında yardım eden veteriner hekimler Altan Kıymaz, Hatice Özbay, biyoloji öğrencileri Irfan Çıldıroğlu, Sibel Çetin, Fatma Eda Arabacı ve bu çalışmayı KÜBAP-01/2012-16 nolu proje kapsamında destekleyen Kastamonu Üniversitesi Rektörlüğü Araştırma Fon Saymanlığına teşekkürlerimi sunarım.

\section{Çıkar Çatışması}

Yazarlar herhangi bir çıkar çatışması bildirmemişlerdir.

\section{KAYNAKLAR}

1. Dehority BA. Rumen ciliate fauna of Alaskan moose (Alces americana), musk-ox (Ovibos moschatus) and Dall mountain sheep (Ovis dalli). J Protozool 1974; 21: 26-32.

2. Imai S. Ciliate protozoa in the rumen of Kenyan zebu cattle, Bos taurus indicus, with the description of four new species. J Protozool 1988; 35: 130-6.

3. Imai S, Han SS, Cheng KJ, Kudo H. Composition of the rumen ciliate population in experimental herds of cattle and sheep in Lethbridge, Alberta, Western Canada. Can J Microbiol 1989; 35: 686-90. [CrossRef]

4. Ito A, Imai S, Ogimoto K. Rumen ciliate composition and diversity of Japanese beef black cattle in comparision with those of holsteinfriesian cattle. J Vet Med Sci 1994; 56: 707-14. [CrossRef]

5. De La Fuente G, Skirnisson K, Dehority BA. Rumen ciliate fauna of Icelandic cattle, sheep, goats and reindeer. Zootaxa 2006; 1377: 47-60. 
6. Imai S. Rumen ciliate protozoal faunae of bali cattle (Bos javanicus domesticus) and water buffalo (Bubalus bubalis) in Indonesia, with the description of a new species, Entodinium javanicum sp. nov. Zool Sci 1985; 2: 591-600

7. Dehority BA. Specificity of rumen ciliate protozoa in cattle and sheep. J Protozool 1978; 25: 509-13.

8. Öktem N, Göçmen B. Türkiye evcil sığır (Bos taurus taurus L.) işkembesinden yenibirsiliyat grubu (Entodiniomorpida: Ophryoscolecidae) ve yeni bir tür Entodinium basoglui sp. nov. Hakkında. Tr J Zool 1996; 20: 271-8.

9. Lynn D. The Ciliated Protozoa, Characterization, Classification and Guide to the Literature. Third Edition. Springer; 2008.

10. Mishima $T$, Katamoto $H$, Horii $Y$, Kakengi VA, Ito A. Rumen ciliates from Tanzanian short zebu cattle, Bos taurus indicus, and the infraciliature of Entodinium palmare n. sp. and Enoplopolastron stokyi (Buisson, 1924). Eur J Protistol 2009; 45: 77-86. [CrossRef]

11. Ito A, Imai S. Ciliated protozoa in the rumen of holstein-friesian cattle (Bos taurus taurus) in Hokkaido, Japan, with the description of two new species. Zool Sci 1990; 7: 449-58.

12. Ogimoto K, Imai S. Atlas of Rumen Microbiology. Japan Scientific Societies Press; 1981.

13. Göçmen B, Gürelli G. Rumen entodiniid ciliated protozoan fauna (Entodiniomorphida: Entodiniidae) of domestic sheep (Ovis ammon aries L.) from Nothern Cyprus, with a description of a new species, Entodinium cypriensis sp. nov. Turk J Zool 2009; 33: 169-80.

14. Göçmen B, Gürelli G. The occurence of the rumen ciliate Entodinium constrictum Dehority, 1974 (Entodiniidae, Entodiniomorphida) from domestic sheep (Ovis ammon aries L.) in Northern Cyprus. NorthWestern J Zool 2009; 5: 301-6.

15. Dogiel VA. Monographie der familie Ophryoscolecidae. Arch F Protistenk 1927; 59: 1-288.

16. Kofoid CA, MacLennan RF. Ciliates from Bos indicus Linn. I. The genus Entodinium Stein. Univ of California Publ in Zool 1930; 33: 471-544.

17. Hsiung TS. A general survey of the protozoan fauna of the rumen of the Chinese cattle. Bull Fan Mem Inst Biol 1932; 3: 87-107.

18. Clarke RTJ. Ciliates of the rumen of domestic cattle (Bos taurus L.). New Zealand J Agric Res 1964; 7: 248-57. [CrossRef]
19. Imai S, Katuno M, Ogimoto K. Distribution of rumen ciliate protozoa in cattle, sheep and goat and experimental transfaunation of them. Jpn J Zootech Sci 1978; 49: 494-505.

20. Imai S, Shimizu M, Kinosita M, Toguchi M, Ishii T, Fujita J. Rumen ciliate protozoal fauna and composition of the cattle in Japan. Bull Nippon Vet Zootech Coll 1982; 31: 70-4.

21. Imai S, Ogimoto K. Parabundleia ruminantium gen.n., sp. n., Diplodinium mahidoli sp. n. with two formae, and Entodinium parvum forma monospinosum forma $\mathrm{n}$. from the Zebu cattle (Bos indicus L., 1758) in Thailand. Jpn J Vet Sci 1983; 45: 585-91. [CrossRef]

22. Imai S. Rumen ciliate protozoal fauna of zebu cattle (Bos taurus indicus) in Sri Lanka, with the description of a new species, Diplodinium sinhalicum sp. nov. Zool Sci 1986; 3: 699-706.

23. Imai S, Han SS, Cheng KJ, Kudo H. Composition of the rumen ciliate population in experimental herds of cattle and sheep in Lethbridge, Alberta, Western Canada. Can J Microbiol 1989; 35: 686-90. [CrossRef]

24. Imai S, Kinoshita M. Comparison of rumen ciliate compositions among hereford, holstein and zebu cattle in Mexico. Rev Soc Mex Hist Nat 1997; 47: 85-91.

25. Göçmen B, Öktem N. New rumen ciliates from Turkish domestic cattle (Bos taurus L.) I. The presence of Entodinium dalli Dehority 1974 with a new forma, E. dalli f. rudidorsospinatum n. f. and comparisons with E. williamsi n. sp. Eur J Protistol 1996; 32: 513-22. [CrossRef]

26. Göçmen B, Tosunoğlu M, Falakalı B. New rumen ciliates from Turkish domestic cattle (Bos taurus L.): 3. Entodinium oektemae n. sp. and Entodinium imaii n. sp. (Entodiniidae, Entodiniomorphida). Turk J Zool 2001; 25: 269-74.

27. Göçmen B, Falakalı Mutaf B, Tosunoğlu M. New rumen ciliates from Turkish domestic cattle (Bos taurus L.): IV. Eudiplodinium dehorityi n. sp. Acta Parasit Turc 2001; 25: 305-7.

28. Göçmen B, Dehority BA, Rastgeldi S. Ciliated protozoa in the rumen of Turkish domestic cattle (Bos taurus L.). J Eukaryot Microbiol 2003; 50: 104-8. [CrossRef]

29. Imai S, Rung G. Ciliate protozoa in the forestomach of the Bactrian camel in Inner-Mongalia, China. Jpn Vet Sci 1990; 52: 1069-72. [CrossRef] 